\title{
ALGEBRAIC SIGNAL PROCESSING THEORY: AN OVERVIEW
}

\author{
Markus Püschel \\ Electrical and Computer Engineering \\ Carnegie Mellon University, Pittsburgh, U.S.A.
}

\begin{abstract}
We give an overview of the algebraic signal processing theory, a recently proposed generalization of linear signal processing (SP). Algebraic SP (ASP) is built axiomatically on top of the concept of a signal model, which is a triple $(\mathcal{A}, \mathcal{M}, \Phi)$, where $\mathcal{A}$ is a chosen algebra of filters, $\mathcal{M}$ an associated $\mathcal{A}$-module of signals, and $\Phi$ generalizes the idea of a $z$-transform. ASP encompasses standard time SP (continuous and discrete, infinite and finite duration), but goes beyond it, for example, by defining meaningful notions of space SP in one and higher dimensions, separable and non-separable. ASP identifies many known transforms as Fourier transforms for a suitably chosen signal model and provides the means to derive and explain existing and novel transform algorithms. As one example, the discrete cosine transform is in ASP the Fourier transform for the finite space model and possesses general radix CooleyTukey type algorithms derived by the theory.
\end{abstract}

\section{INTRODUCTION}

The goal of this paper is to give a short introduction to the algebraic signal processing (ASP) theory that was recently developed in a series of papers $[1,2,3,4,5,6,7,8,9]$. ASP is a novel approach and generalization of linear signal processing (SP). Algebra here refers to the theory of groups, rings, and fields, while linear SP refers to SP built around a collection of concepts including signals as elements of vector spaces, filters as linear operators on signals, $z$-transform, spectrum, and Fourier transform. ASP generalizes these concepts and thus linear SP, to provide a unifying framework for many existing SP methods. Further, ASP enables the derivation of new SP methods including new notions of $z$-transforms, Fourier transforms, and novel fast transform algorithms in one and higher dimensions, separable and non-separable.

These advances are made possible through the connection between SP and algebra that we reveal and explore. Algebra has to date been only sparsely used in mainstream SP. In the adjacent fields of system theory and communications, algebra is a well-established part of the theoretical foundation.

The focus of this paper is on conveying the ideas and showing some of the results obtained so far, rather than on a rigorous development. Also, we do not give a detailed comparison to previous work on algebra in signal processing and mathematics, which is provided in the references above. Here we only give to examples that are related to ASP. First, the algebraic methods developed in SP to derive fast Fourier transform algorithms, most notably [10]. Second, the work in mathematics on Fourier transforms on groups; see [11] for an overview.

Organization. Section 2 explains why algebra naturally describes the structure in SP and gives details on the general foundation of ASP, most importantly, the concept of a signal model on which ASP is built. In Section 3, we then provide

This work was supported by NSF award 0310941. several examples of signal models for time and space, finite and finite, separable and non-separable SP. Continuous signal models and first ideas on general sampling are provided in Section 4. Section 5 briefly reviews a major application of ASP: the discovery, concise derivation, and classification of fast transform algorithms. Finally, we offer conclusions in Section 6.

\section{ASP: MOTIVATION AND FOUNDATION}

We focus on discrete SP and on complex signals, but ASP extends to continuous SP and to other basefields. We repeat that ASP is a general approach to linear SP, henceforth simply called SP.

Motivation for ASP: SP is algebraic. The key observation underlying ASP is that standard SP is already algebraic in nature. Namely, while the set of filters is usually assumed to be a vector space (addition = parallel connection, scalar multiplication $=$ amplification), it also offers multiplication (serial connection), an operation outside the vector space framework. Thus, algebraically, the filter space is more than a vector space: it is an algebra ${ }^{1} \mathcal{A}$. In SP, the set of signals is also typically assumed to be a vector space, and filters are linear operators on this space. Algebraically, this means that the signal space is an $\mathcal{A}$-module $\mathcal{M}$. As an example, in infinite discrete-time SP, usually: ${ }^{2}$

$$
\begin{aligned}
& \mathcal{A}=\left\{h=\sum_{n \in \mathbb{Z}} h_{n} x^{n} \mid \mathbf{h}=\left(h_{n}\right)_{n \in \mathbb{Z}} \in \ell^{1}(\mathbb{Z})\right\}, \\
& \mathcal{M}=\left\{s=\sum_{n \in \mathbb{Z}} s_{n} x^{n} \mid \mathbf{s}=\left(s_{n}\right)_{n \in \mathbb{Z}} \in \ell^{2}(\mathbb{Z})\right\} .
\end{aligned}
$$

In the above, the filters $h$ and signals $s$ are expressed after applying the $z$-transform; we set $x=z^{-1}$ for simplicity and consistency with later developments. Note that coordinate sequences (sequences of numbers) $\mathbf{h}$ and $\mathbf{s}$ are written bold-faced, whereas elements of algebras and modules are not.

The above discussion naturally places SP into the context of module theory or representation theory of algebras, a welldeveloped mathematical discipline (see [12], for example).

The foundation of ASP: Signal model. ASP is built on top of a concept we call a signal model, defined as a triple $(\mathcal{A}, \mathcal{M}, \Phi)$, where $\mathcal{A}$ is a chosen filter algebra, $\mathcal{M}$ an associated $\mathcal{A}$-module of signals, and $\Phi$ a bijective mapping from a vector space $V \leq \mathbb{C}^{I}$ of signal values ${ }^{3}$ over some index domain $I$ to $\mathcal{M}$. $\Phi$ is best explained through an example: With $\mathcal{M}$ as in (2),

$$
\Phi: \ell^{2}(\mathbb{Z}) \rightarrow \mathcal{M}, \quad \mathbf{s} \mapsto \sum_{n \in \mathbb{Z}} s_{n} x^{n},
$$

\footnotetext{
${ }^{1}$ An algebra is a vector space that is also a ring.

${ }^{2}$ Note that allowing $\ell^{2}$ coefficients for filters would destroy the algebra property; the serial connection of two $\ell^{2}$ filters is in general not an $\ell^{2}$ filter.

${ }^{3} V \leq \mathbb{C}^{I}$ means that $V$ is a subspace of $\mathbb{C}^{I}$.
} 
is the well-known $z$-transform. The signal model $(\mathcal{A}, \mathcal{M}, \Phi)$ defined through (1)-(3) is the one commonly adopted for infinite discrete-time SP. The mapping $\Phi$ makes filtering available in $V$ and defines it through the multiplication of filters $h \in \mathcal{A}$ with signals $s \in \mathcal{M}$.

We state the key point in ASP, which also motivates the definition of a signal model: If a signal model $(\mathcal{A}, \mathcal{M}, \Phi)$ is given, all the basic ingredients for SP are automatically defined (if they exist) and provided by the representation theory of algebra.

What this means is that different signal models have different notions of filtering or convolution, spectrum, and Fourier transform, among others. This is well-known for continuoustime and discrete-time, infinite and finite (duration) SP. For example, for periodic discrete-time signals (or signals living on a finite interval periodically extended), SP defines a special form of filtering called circular convolution, since the result of applying the usual convolution defined for infinitely-supported sequence to such signals would result in a signal which would no longer be periodic (or living on the same finite interval). In SP, it is also well known, that in such a case, the appropriate Fourier transform to use is the discrete Fourier transform applied to a period (a finite number of points). However, as we will demonstrate, many other signal models are possible and reasonable, leading to different notions of filtering and Fourier transform that are more appropriate for certain applications. One such example is the DCT, which, as we show later, is a Fourier transform for a specific signal model appropriate for finite "space" signals, for which filtering is defined appropriately.

Shift-invariance and polynomial algebras. At this point we have many different options on how to do SP arising from the many possible choices of a signal model. The question is, which models are the most relevant for applications? To answer this question, we first need the notion of shift-invariance. Shifts are the generators of $\mathcal{A}$, i.e., every element in $\mathcal{A}$ is a linear combination of powers of shifts. For example, $\mathcal{A}$ in (1) has one shift $x=z^{-1}$ only since it is a 1-D model. A signal model is shift-invariant if shifts $\in \mathcal{A}$ and filters $h \in \mathcal{A}$ commute. We achieve this by requiring $\mathcal{A}$ to be commutative (e.g., $\mathcal{A}$ in (1) is commutative as expected). For discrete signal models, we can classify the possible commutative algebras:

- Infinite-dimensional commutative algebras $\mathcal{A}$ are spaces of series in one or more variables (as in (1)).

- Finite-dimensional commutative algebras $\mathcal{A}$ are polynomial algebras in one or more variables. In one variable (i.e., with one shift), they are written as

$$
\mathcal{A}=\mathbb{C}[x] / p(x)=\{q(x) \mid \operatorname{deg} q<\operatorname{deg} p\},
$$

where $p(x)$ is a fixed polynomial of degree $n$. In words, $\mathbb{C}[x] / p(x)$ is the set of polynomials of degree less than $n$; addition and multiplication are defined modulo the fixed polynomial $p(x)$. In higher dimensions, more shifts are available, which leads to polynomial algebras in more variables.

Generalization of SP Concepts. Above we alluded that ASP generalizes the fundamental concepts in SP, such as filtering and Fourier transform. This is done by translating the concepts into the language of algebra, which makes them in a sense "portable" across different signal models. Then the general concepts can be instantiated for important special cases.
We illustrate this approach in Table 1. Columns one and two translated some basic SP concepts into algebra. The last column is the special case of a shift-invariant finite 1-D model.

Our starting point is the core concept of a signal model $(\mathcal{A}, \mathcal{M}, \Phi)$. The reader may keep the "prototype signal model" of discrete time in mind (given by (1),(2), and (3)). We discuss columns one and two of Table 1 . Filters are elements of $\mathcal{A}$, while signals are elements of $\mathcal{M}$ expressed in the basis implicitly chosen by $\Phi\left(x^{n}=z^{-n}\right.$ in discrete time). Filtering is the (given) operation of $\mathcal{A}$ on the module $\mathcal{M}$. Impulses are the basis vectors of $\mathcal{M}$, and filtering those gives the impulse response of that filter. For the Fourier transform, one first has to identify all irreducible ("smallest") $\mathcal{A}$-submodules $\mathcal{M}_{\omega}$ of $\mathcal{M}$. In discrete time, each $\mathcal{M}_{\omega}$ is spanned by one element: $\sum e^{j \omega n} x^{n}$ for $\omega \in[0,2 \pi)$, which is an eigenfunction for all filters in $\mathcal{A}$. In general, the dimension of $\mathcal{M}_{\omega}$ may be larger than one, and the domain of the index $\omega$ may be continuous, discrete, infinite, or finite. The Fourier transform is now the collection of projections onto these submodules.

Above, we asserted that if the signal model supports shiftinvariant $\mathrm{SP}$ and is for finite discrete signals $\mathbf{s} \in \mathbb{C}^{n}$, then the filter algebra $\mathcal{A}=\mathbb{C}[x] / p(x)$ is a polynomial algebra (see (4)). This is an important special case of the general theory and is shown in the last column of Table 1 . We assume $\mathcal{M}=\mathcal{A}$ and $\Phi$ as given in the caption. Filtering in this model is the multiplication of the polynomials $h(x) \in \mathcal{A}$ and $s(x) \in \mathcal{M}$ modulo the fixed polynomial $p(x)$, and the Fourier transform $\Delta$ is given by the Chinese remainder theorem. ${ }^{4}$ In fact, $\Delta$, as a linear mapping, can in this case be expressed as a matrix containing the evaluations of all basis polynomials in the basis $b=\left(p_{0}, \ldots, p_{n-1}\right)(n=\operatorname{deg}(p))$ at all zeros $\alpha_{\ell}$ of $p$ :

$$
\Delta \leftrightarrow \mathcal{P}_{b, \alpha}=\left[p_{k}\left(\alpha_{\ell}\right)\right]_{0 \leq \ell, k<n} .
$$

For the finite models shown later in Table 3, this construction connects the polynomial algebras to the actual transforms. For example, for the DFT, $p(x)=x^{n}-1, p_{k}(x)=x^{k}$, $\alpha_{\ell}=$ $\exp (-2 \pi j \ell / n)$, and thus

$$
\mathcal{P}_{b, \alpha}=\mathrm{DFT}_{n}=[\exp (-2 \pi j k \ell / n)]_{0 \leq \ell, k<n} .
$$

\section{EXAMPLES OF SIGNAL MODELS}

Now we are ready to provide examples of signal models, for infinite and finite (duration) signals, for 1-D and 2-D, separable and nonseparable. Some are explicitly used in SP, such as the infinite discrete-time model discussed above; some are implicitly used and could be argued as missing in the current SP theory (for example, we provide the signal model that has the DCT as its Fourier transform); others are novel.

Infinite shift-invariant 1-D models. Table 2 shows three signal models. The first is the standard time model introduced before. The basis $\left(x^{n} \mid n \in \mathbb{Z}\right)$ of $\mathcal{M}$ provides the time structure, that is, the directed operation of the shift $x$ which captures the notion of direction (from left to right) and passage of time (past, present and future). The expression $x \cdot x^{n}=x^{n+1}$ implies that applying the shift $x$ to a point at time $n$ denoted by the basis element $x^{n}$ moves that point to a point at time $(n+1)$ denoted by the basis element $x^{n+1}$. We visualize this operation by the graph in Fig. 2(a), which, as time, is directed. (Visualization is an important concept in ASP: it shows the structure imposed on a signal by the model.)

\footnotetext{
${ }^{4}$ We assume that $p(x)$ has no multiple zeros.
} 


\begin{tabular}{lll}
\hline SP concept & in ASP & shift-invariant finite 1-D model \\
\hline filter & $h \in \mathcal{A}$ (algebra) & $h(x) \in \mathbb{C}[x] / p(x)$ \\
signal & $s=\sum s_{i} b_{i} \in \mathcal{M}(\mathcal{A}$-module) & $s(x)=\sum_{0 \leq k<n} s_{k} p_{k}(x) \in \mathcal{M}$ \\
filtering & $h \cdot s$ & $h(x) s(x) \bmod p(x)$ \\
impulse & basis vector $b_{k} \in \mathcal{M}$ & basis polynomial $p_{k}(x) \in \mathcal{M}$ \\
impulse response of $h \in \mathcal{A}$ & $h \cdot b_{k} \in \mathcal{M}$ & $h(x) p_{k}(x) \bmod p(x)$ \\
Fourier transform & $\Delta: \mathcal{M} \rightarrow \bigoplus_{\omega \in W} \mathcal{M}_{\omega}$ & $\Delta: \mathbb{C}[x] / p(x) \rightarrow \bigoplus_{0 \leq \ell<n} \mathbb{C}[x] /\left(x-\alpha_{\ell}\right)$ \\
& $\left(\mathcal{M}_{\omega}\right.$ irreducible submodules of $\left.\mathcal{A}\right)$ & $\left(\right.$ Chinese remainder theorem, $\alpha_{\ell}$ zero of of $\left.p(x)\right)$ \\
spectrum of signal & $\Delta(s)=\left(s_{\omega}\right)_{\omega \in W}=\omega \mapsto s_{\omega}$ & $\Delta(s(x))=\left(s\left(\alpha_{0}\right), \ldots, s\left(\alpha_{n-1}\right)\right)$ \\
\hline
\end{tabular}

Table 1. Correspondence between SP concepts and algebraic concepts, given a (discrete) signal model $(\mathcal{A}, \mathcal{M}, \Phi)$. The last column shows the special case of a shift-invariant finite 1-D model: $\mathcal{A}=\mathcal{M}=\mathbb{C}[x] / p(x), \Phi: \mathbf{s} \mapsto \sum_{0 \leq k<n} s_{k} p_{k}(x)$. Note that $\Phi$ implicitly chooses a basis in $\mathcal{M}$.

\begin{tabular}{llll}
\hline Concept & Time $\left(x=z^{-1}\right)$ & Space & Generalized space \\
\hline $\mathcal{A}$ & $\left\{\sum_{n \in \mathbb{Z}} h_{n} x^{n}\right\}$ & $\left\{\sum_{n \geq 0} h_{n} T_{n}(x)\right\}$ & $\left\{\sum_{n \geq 0} h_{n} Q_{n}(x)\right\}$ \\
$\mathcal{M}$ & $\left\{\sum_{n \in \mathbb{Z}} s_{n} x^{n}\right\}$ & $\left\{\sum_{n \geq 0} s_{n} C_{n}(x)\right\}$ & $\left\{\sum_{n \geq 0} s_{n} P_{n}(x)\right\}$ \\
$\Phi$ & $z$-transform & $C$-transform $(C \in\{T, U, V, W\})$ & $P$-transform \\
shift $x$ & $x \cdot x^{n}=x^{n+1}$ & $x \cdot C_{n}=\frac{1}{2}\left(C_{n-1}+C_{n+1}\right)$ & $x \cdot P_{n}=\alpha_{n} P_{n-1}+\beta_{n} P_{n}+\gamma_{n} P_{n+1}$ \\
& (time shift) & (space shift) & (general space shift) \\
$\mathcal{F}$ & DTFT & DSFT & $?$ \\
Visualization & Fig. 1(a) & Fig. 1(b) & not shown \\
\hline
\end{tabular}

Table 2. Infinite discrete shift-invariant 1-D signal models $(\mathcal{A}, \mathcal{M}, \Phi)$, associated shift operation, and Fourier transform $\mathcal{F}$. The space model as well as the general space model are novel.

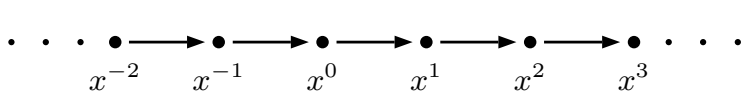

(a) infinite time model

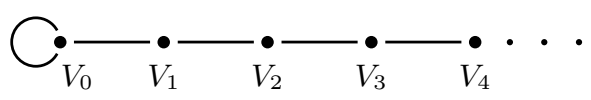

(b) infinite space model (for $C=V$ )

Figure 1. Visualization of the infinite time and space models.

The second model is novel. We call it a space model, which means it has an undirected visualization. Intuitively, undirected models should work better for signals without inherent direction, such as images. Indeed, the finite space models introduced below have the 8 DCTs and 8 DSTs [13] as their Fourier transforms. The undirected structure is achieved by changing the shift operation and thus the basis of the signal module $\mathcal{M}$ as shown in the table. The shift is again denoted by $x$ and we assume that the basis elements in $\mathcal{M}$ are denoted by $C_{n}$. Then we define the "space" shift operation as moving the point $n$ denoted by $C_{n}$ half to the left, to the point $(n-1)$ denoted by $C_{n-1}$ and half to the right, to the point $(n+1)$ denoted by $C_{n+1}$. Solving for $C_{n}$, which are still unknown, we find that the polynomials satisfying $x C_{n}=\frac{1}{2}\left(C_{n-1}+C_{n+1}\right)$ are exactly the (general) Chebyshev polynomials [14]. Four special cases $C \in\{T, U, V, W\}^{5}$ are important: For example,

\footnotetext{
${ }^{5}$ These are called Chebyshev polynomials of the first, second, third
}

for $C=V, V_{0}=1, V_{1}=2 x-1, V_{2}=4 x^{2}-2 x-1, \ldots$ and $V_{-n}=V_{n-1}$. This means the left half depends on the right half, and the model is for unilateral signals only. The model for $C=V$ is visualized in Fig. 2(b). The graph is undirected and the extra edge from $V_{0}$ to itself is due to the boundary condition $V_{-1}=V_{0}$. The other three types of Chebyshev polynomials yield other boundary conditions.

Filtering in the space model is the multiplication of series $s \in \mathcal{M}$ with $h \in \mathcal{A}$ using the distributivity law and the property $T_{n} C_{k}=\frac{1}{2}\left(C_{n-k}+C_{n+k}\right)$.

The third model (last column in Table 2) shows that with ASP it is possible to build a meaningful SP framework for more general shifts. In this case the $P_{n}$ become arbitrary orthogonal polynomials [15].

Finite shift-invariant 1-D models. A strength of ASP is that it provides a comprehensive framework for finite signals. In fact, we have identified signal models (and thus the proper notions of " $z$-transform," convolution, spectrum etc.), for practically all trigonometric transforms [3, 2]. All these models are provided by polynomial algebras since they are shift-invariant. We give a few examples in Table 3 .

The finite time model (finite number of signal values with circular convolution) is associated with $\mathbb{C}[x] /\left(x^{n}-1\right)$, that is, filtering is the multiplication of polynomials modulo $p(x)=$ $x^{n}-1$, which is equivalent to circular convolution, a wellknown fact [10]. The notion of finite $z$-transform is novel. Note that in $\mathcal{M}, x^{n}-1=0$ or $x^{n}=1$ holds, which encodes and fourth kind, respectively. 


\begin{tabular}{llll}
\hline Concept & Time & Space (16 cases) & Space (special case) \\
\hline $\mathcal{A}=\mathcal{M}$ & $\mathbb{C}[x] /\left(x^{n}-1\right)$ & $\mathbb{C}[x] / p(x)$ & $\mathbb{C}[x] /\left(V_{n}-V_{n-1}\right)$ \\
$\Phi$ & $\mathbf{s} \mapsto \sum_{0 \leq k<n} s_{k} x^{k}$ & $\mathbf{s} \mapsto s_{k} C_{k}(x)$ & $\mathbf{s} \mapsto s_{k} V_{k}(x)$ \\
& (finite $z$-transform) & (finite $C$-transform, $C \in\{T, U, V, W\})$ & (finite $V$-transform) \\
$\mathcal{F}$ & DFT & 16 DCTs/DSTs & DCT, type 2 \\
Visualization & Fig. 2(a) & not shown & Fig. 2(b) \\
\hline
\end{tabular}

Table 3. Finite discrete shift-invariant 1-D signal models $(\mathcal{A}, \mathcal{M}, \Phi)$.

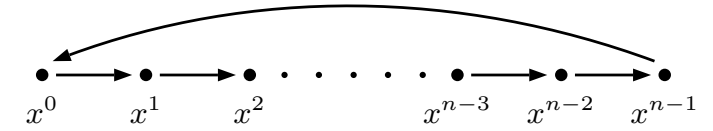

(a) finite time model (associated with $\mathrm{DFT}_{n}$ )

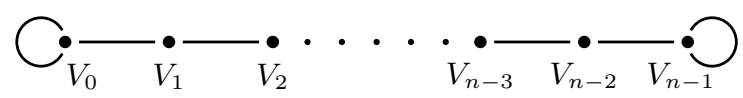

(b) finite space model (associated with DCT $-2_{n}$ )

Figure 2. Visualization of finite time and space models.

the cyclic boundary condition and yields the visualization as directed circle. In words, applying the DFT assumes the signal to be associated with the nodes of a directed circle (equivalent to being periodic). Time models with different boundary conditions can be built (by changing $p(x)=x^{n}-1$ but keeping the basis).

The finite space models associated with the 16 DCTs and DSTs [13] are only sketched; $p(x)$ depends on the transform. The model that has "the" DCT (that is, type 2) as Fourier transform is shown in the last column. The visualizations are all undirected line graphs but differ in the boundary conditions.

A generalized finite space model (corresponding to the last column of Table 2) can also be built, as well as the models corresponding to the various versions of real DFTs and Hartley transforms [2].

Finite shift-invariant 2-D models. The above discussion, and thus ASP, generalizes to higher dimensions by allowing more than one shift operator. We give examples for the finite case, where we get polynomial algebras in at least two variables. Four models are visualized (without boundary conditions) in Fig. 3, shown with their associated Fourier transform. The first two are separable, the last two are novel nonseparable space models $[6,8]$.

As examples, the signal models underlying the 2-D DFT and the DTT (discrete triangle transform) are, respectively:

$$
\begin{gathered}
\mathcal{A}=\mathcal{M}=\mathbb{C}[x, y] /\left\langle x^{n}-1, y^{n}-1\right\rangle, \\
\Phi: \mathbf{s} \mapsto \sum_{0 \leq k, \ell<n} s_{k, \ell} x^{k} y^{\ell} ; \\
\mathcal{A}=\mathcal{M}=\mathbb{C}[x, y] /\left\langle C_{0, n}(x, y), C_{n, 0}(x, y)\right\rangle, \\
\Phi: \mathbf{s} \mapsto \sum_{0 \leq k, \ell<n} s_{k, \ell} C_{k, \ell}(x, y) .
\end{gathered}
$$

In (6), $C_{k, \ell}(x, y)$ are the the little-known Chebyshev polynomials in two variables [16].

Nonseparable directed SP in two and higher dimensions has been developed in the work of Mersereau et al. [17, 18].
Using ASP, a similar theory of undirected or spatial SP is possible including further generalizations (for example, the last column of Table 2).

\section{CONTINUOUS MODELS AND SAMPLING}

ASP encompasses continuous and discrete SP. A particular task is to identify the continuous signal models underlying the discrete ones via sampling. We briefly discuss the situation in 1-D time and 1-D space.

1-D time. In 1-D time SP, it is standard to consider four cases of signal models visualized in Figure 4. The discrete ones (bottom row) were discussed before. The infinite continuous model (top left) is for $V=\mathcal{L}^{2}(\mathbb{R})$ (viewed as vector space) and given by $\mathcal{A}=\mathcal{L}^{1}(\mathbb{R}), \mathcal{M}=\mathcal{L}^{2}(\mathbb{R})$ with the standard convolution $h \star s(t)=\int h(\tau) s(t-\tau) d \tau$, and $\Phi$ is the identity mapping. ${ }^{6}$ The finite continuous model (top right) is more interesting. It is for the vector space $V=\mathcal{L}^{2}[0, I]$, and given by $\mathcal{A}=\mathcal{L}^{1}(C), \mathcal{M}=\mathcal{L}^{2}(C)$, where $C=\mathbb{R} / I \mathbb{Z}$ is the circle with a circumference of length $I . \Phi$ is the natural mapping from $V$ onto $\mathcal{M}$, that is, it extends a signal on $[0, I]$ periodically. Thus, the visualization is a directed circle. The sampling theorems associated with Figure 4 are well known.

1-D space. The corresponding situation for the DCT, type 2 , is shown in Figure 5. The small circles in the visualizations signify a symmetric extension. The discrete models were discussed before. The key in these models is to start with a different notion of convolution in the infinite continuous case, namely with

$$
h \star s(t)=\int h(\tau)\left(\frac{1}{2}(s(t-\tau)+s(t+\tau)) d \tau .\right.
$$

This definition makes sense only for right-sided filters and signals, considered to be symmetrically extended to the left. So the signal model is for $V=\mathcal{L}^{2}\left(\mathbb{R}^{+}\right)$, and $\mathcal{A}=\mathcal{L}^{1}(S)$, $\mathcal{M}=\mathcal{L}^{2}(S)$, where $S$ is the structure shown in Figure 5 (top left). $\Phi$ is the natural mapping from $V$ onto $\mathcal{M}$, that is, $\Phi$ extends $s \in V$ symmetrically. One can easily verify that (7) is well-defined on $S$.

The eigenfunctions for (7) are $\cos (\omega t), \omega \in[0, \pi]$, which yields the Fourier transform $S(\omega)=\int s(t) \cos (\omega t) d t$ for the continuous space model.

In the finite continuous case (top right in Figure 5), the signal module consists of $\mathcal{L}^{2}$ functions on a symmetrically extended interval of length $I$, visualized as shown.

We derived a sampling theorem for the right column in Figure 5 in [9]. A complete treatment is in preparation [19].

\footnotetext{
${ }^{6}$ The only thing $\Phi$ does is change the viewpoint: $s \in \mathcal{L}^{2}(\mathbb{R})$ is viewed as an element of a vector space, whereas $\Phi(s)$ is an element of a module. This is somewhat different from the $z$-transform, but seems to make the most sense in the current state of this research.
} 


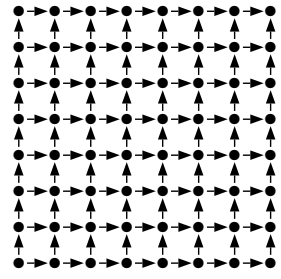

(a) 2-D DFT

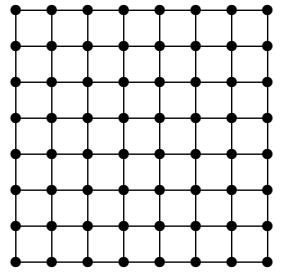

(b) 2-D DCT/DST

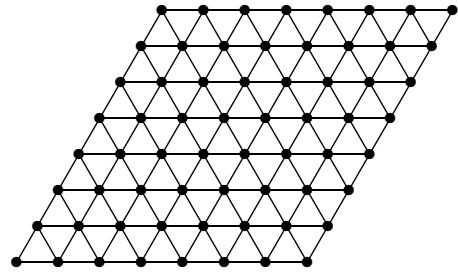

(c) DTT

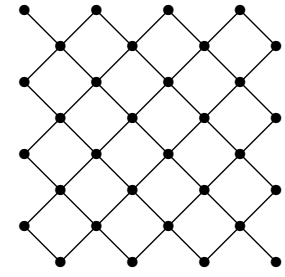

(d) DQT

Figure 3. Visualizations of 2-D signal models (without boundary conditions) underlying the transform in the subtitle. (a) is a time model, (b)-(d) are space models; (c) and (d) are nonseparable.
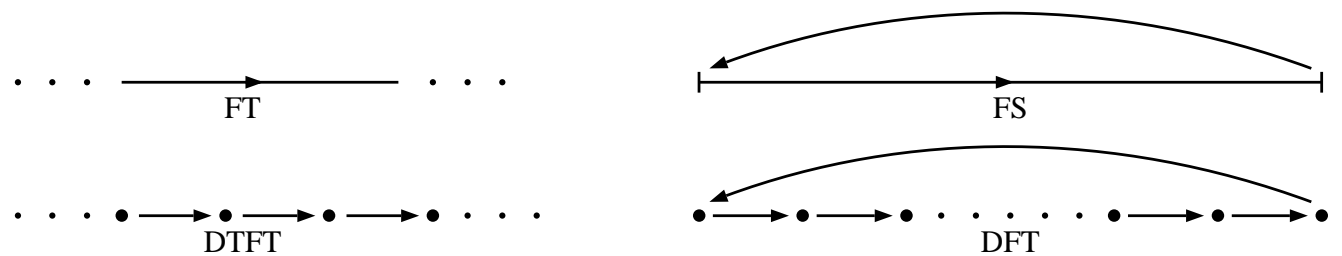

Figure 4. Standard continuous (top) and discrete (bottom), infinite (left) and finite (periodically extended, right) 1-D time SP including the associated Fourier transforms. All the signal models are directed. The bottom row is obtained through sampling the top row. The right column is obtained by sampling the spectrum of the left column.

\section{FAST TRANSFORM ALGORITHMS}

One crucial application of ASP is in the discovery, derivation, and classification of transform algorithms. There are more than 100 publications on this topic, but with few exceptions their derivation is based on manipulating the transform coefficients, giving no insight into the structure of these algorithms nor reasons for their existence. Most importantly, it is not clear whether all important algorithms have been found.

Using ASP, we have identified a few principles that account for practically all transform algorithms and also found many new ones. This was possible by working with the signal model, or polynomial algebra, underlying the transform. For example, Table 4 shows general-radix Cooley-Tukey type algorithms for the DFT, real DFT, and all 16 DCTs/DSTs in Kronecker product notation $[20,21] .^{7}$ All these algorithms are based on only one general theorem; thus, the similarities in their structure.

Namely, assume that a 1-D transform is a Fourier transform for a signal model of the form in the last column in Table 1 and assume that the polynomials $p(x)=q(r(x))$ decomposes. ${ }^{8}$ Then, a stepwise application of the Chinese remainder theorem yields the following result. For full details see $[4,22,23]$.

Theorem 1 (General Cooley-Tukey Type Algorithms) We use previous notation and assume that $p(x)=q(r(x))$. Then

$$
\mathcal{P}_{b, \alpha}=P\left(\bigoplus_{0 \leq i<k} \mathcal{P}_{d, \alpha_{i}^{\prime}}\right)\left(\mathcal{P}_{c, \beta} \otimes I_{m}\right) B
$$

where $B$ is a base change matrix and $P$ a permutation matrix.

\footnotetext{
${ }^{7}$ The exact form of all the matrices is not of importance here and is omitted due to lack of space.

${ }^{8}$ Which is different from a factorization.
}

The algorithm derivation generalizes to signal models in higher dimensions such as those in (5) and (6) and Fig. 3 (see, for example, [7]). Similar theorems generalize split-radix and Rader type algorithms (see [22] for first results).

\section{CONCLUSIONS}

We hope we could convey the scope and the relevance of ASP, which is work in progress. To summarize:

- ASP aims to be a general, consistent, axiomatic framework for SP with many instantiations that include but go beyond standard time (directed) SP.

- In particular, ASP generalizes many concepts that are fundamental to SP including filtering/convolution, $z$-transform, and Fourier transform. Many existing transforms become Fourier transforms for suitable signal models in ASP and thus have associated convolutions and " $z$-transforms."

- By bringing algebra into SP, ASP offers a new set of tools. Examples include a comprehensive theory of transform algorithms and new signal models and transforms for nonseparable 2-D SP.

- Shift-invariance simplifies the algebra used to working with series and polynomials only. This makes ASP accessible without special algebra education.

ASP provides insights and results beyond the above. For details we refer to the cited papers.

\section{REFERENCES}

[1] M. Püschel and J. M. F. Moura, "The algebraic structure in signal processing: Time and space," in Proc. ICASSP, 2006.

[2] M. Püschel and J. M. F. Moura, "Algebraic signal processing theory: Foundation and 1-D time," submitted for publication.

[3] M. Püschel and J. M. F. Moura, "Algebraic signal processing theory: 1-D space," submitted for publication. 

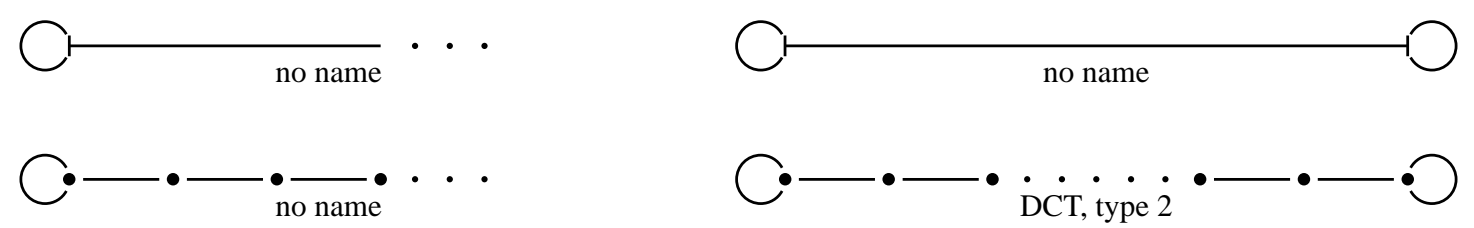

Figure 5. Situation for 1-D space SP, analogous to Table 4, and underlying the DCT, type 2 . All the signal models are undirected. The associated Fourier transforms have no specific names.

$$
\begin{aligned}
& \mathrm{DFT}_{n}=L_{m}^{n}\left(I_{k} \otimes \mathrm{DFT}_{m}\right) T_{m}^{n}\left(\mathrm{DFT}_{k} \otimes I_{m}\right) \\
& \left.\operatorname{RDFT}_{k m}=P_{m}^{k m}\left(\operatorname{RDFT}_{m} \oplus\left(\bigoplus_{1 \leq i<k / 2} \operatorname{rDFT}_{2 m}(i / k)\right) \oplus \operatorname{RDFT}_{3}\right)_{m}\right)\left(\operatorname{RDFT}_{k} \otimes I_{m}\right) \\
& \operatorname{rDFT}_{2 k m}(u)=\left(K_{m}^{k m} \otimes I_{2}\right)\left(\bigoplus_{0 \leq i<k} \operatorname{rDFT}_{2 m}(U(k, i, u))\right)\left(\operatorname{rDFT}_{2 k}(u) \otimes I_{m}\right) \\
& \mathrm{T}_{n}=K_{m}^{n}\left(\bigoplus_{i} \mathrm{~T}_{m}\left(\frac{i+1 / 2}{k}\right)\right)\left(\mathrm{DCT}-3_{k} \otimes I_{m}\right) B_{n, k}^{(*)}, \quad \mathrm{T} \in\{\mathrm{DCT}-3 / 4, \mathrm{DST}-3 / 4\} \\
& \mathrm{T}_{n}=P_{k, m}^{(*)}\left(\mathrm{T}_{m} \oplus\left(\bigoplus_{i} \mathrm{~T}_{m}\left(\frac{i}{k}\right)\right)\left(\overline{\mathrm{DST}-1}_{k-1} \otimes I_{m}\right)\right) B_{k, m}^{(*)}, \quad \mathrm{T} \in\{\mathrm{DCT}-1 / 2, \mathrm{DST}-1 / 2\} \\
& \mathrm{T}_{n}=P_{k, m}^{(*)}\left(\left(\bigoplus_{i} \mathrm{~T}_{2 m+1}\left(\frac{2 i+1}{k}\right)\right)\left(\overline{\mathrm{DST}-5}_{(k-1) / 2} \otimes I_{2 m+1}\right) \oplus \mathrm{T}_{m}\right) B_{k, m}^{(*)}, \quad \mathrm{T} \in\{\mathrm{DCT}-5 / 6, \mathrm{DST}-5 / 6\} \\
& \mathrm{T}_{n}=P_{k, m}^{(*)}\left(\left(\bigoplus_{i} \mathrm{~T}_{2 m+1}\left(\frac{2 i+1}{k}\right)\right)\left(\overline{\mathrm{DST}-7}_{(k-1) / 2} \otimes I_{2 m+1}\right) \oplus \mathrm{T}_{m}\right) B_{k, m}^{(*)}, \quad \mathrm{T} \in\{\mathrm{DCT}-7 / 8, \mathrm{DST}-7 / 8\}
\end{aligned}
$$

Table 4. General radix Cooley-Tukey type algorithms for the DFT, real DFT (RDFT), the auxiliary transform rDFT, and the 16 types of DCTs and DSTs, organized into four groups. $L, K, P$ are permutations, and $B$ is sparse. $P, B$ are different for different transforms. All algorithms are based on Theorem 1.

[4] M. Püschel and J. M. F. Moura, "Algebraic signal processing theory: Cooley-Tukey type algorithms for DCTs and DSTs," submitted for publication.

[5] M. Püschel and M. Rötteler, "Algebraic signal processing theory: 2-D spatial hexagonal spatial lattice," submitted for publication.

[6] M. Püschel and M. Rötteler, "The discrete triangle transform," in Proc. ICASSP, 2004, vol. 3, pp. 45-48.

[7] M. Püschel and M. Rötteler, "Cooley-Tukey FFT like algorithm for the discrete triangle transform," in Proc. 11th IEEE DSP Workshop, 2004, pp. 158-162.

[8] M. Püschel and M. Rötteler, "Fourier transform for the spatial quincunx lattice," in Proc. ICIP, 2005.

[9] J. Kovacevic and M. Püschel, "Sampling theorem associated with the discrete cosine transform," in Proc. ICASSP, 2006.

[10] H. J. Nussbaumer, Fast Fourier Transformation and Convolution Algorithms, Springer, 2nd edition, 1982.

[11] D. Maslen and D. Rockmore, "Generalized FFTs - a survey of some recent results," in Proc. IMACS Workshop in Groups and Computation, 1995, vol. 28, pp. 182-238.

[12] W. C. Curtis and I. Reiner, Representation Theory of Finite Groups and Associative Algebras, Interscience, 1962.

[13] Z. Wang and B. R. Hunt, "The discrete W transform," Applied Mathematics and Computation, vol. 16, pp. 1948, 1985.
[14] T. J. Rivlin, The Chebyshev Polynomials, Wiley Interscience, 1974

[15] G. Szegö, Orthogonal Polynomials, AMS Colloquium Publications, 3rd edition, 1967.

[16] T. Koornwinder, "Orthogonal polynomials in two variables which are eigenfunctions of two algebraically independent partial differential operators (part III)," Indag. Math., vol. 36, pp. 357-369, 1974.

[17] D. E. Dudgeon and R. M. Mersereau, Multidimensional Digital Signal Processing, Prentice-Hall, 1984.

[18] R. M. Mersereau, "The processing of hexagonally sampled two-dimensional signals," Proceedings of the IEEE, vol. 67, no. 6, pp. 930-949, 1979.

[19] J. Kovacevic and M. Püschel, "Algebraic signal processing theory: Sampling in 1-D space," in preparation.

[20] R. Tolimieri, M. An, and C. Lu, Algorithms for Discrete Fourier Transforms and Convolution, Springer, 2nd edition, 1997.

[21] C. Van Loan, Computational Framework of the Fast Fourier Transform, Siam, 1992.

[22] M. Püschel and J. M. F. Moura, “The algebraic approach to the discrete cosine and sine transforms and their fast algorithms," SIAM Journal of Computing, vol. 32, no. 5, pp. 1280-1316, 2003.

[23] Y. Voronenko and M. Püschel, "Algebraic derivation of general radix Cooley-Tukey algorithms for the real discrete Fourier transform," in Proc. ICASSP, 2006. 